\title{
Fuzzy C Mean Thresholding based Level Set for Automated Segmentation of Skin Lesions
}

\author{
Ammara Masood, Adel Ali Al-Jumaily \\ School of Electrical, Mechanical and Mechatronic Engineering, University of Technology, Sydney, Australia. \\ Email: ammara.masood@student.uts.edu.au,Adel.Al-Jumaily@uts.edu.au
}

Received April, 2013.

\begin{abstract}
Accurate segmentation is an important and challenging task in any computer vision system. It also plays a vital role in computerized analysis of skin lesion images. This paper presents a new segmentation method that combines the advantages of fuzzy C mean algorithm, thresholding and level set method. 3-class Fuzzy C mean thresholding is applied to initialize level set automatically and also for estimating controlling parameters for level set evolution. Parameters for performance evaluation are presented and segmentation results are compared with some other state-of-the-art segmentation methods. Increased true detection rate and reduced false positive and false negative errors confirm the effectiveness of proposed method for skin cancer detection.
\end{abstract}

Keywords: Skin Cancer; Segmentation; Diagnosis; Fuzzy; Thresholding; Level Sets

\section{Introduction}

The incidence of skin cancer is rapidly increasing through-out the world [1]. An estimated 76,250 new cases of invasive melanoma were diagnosed in the US in 2012, with an estimated 9,180 resulting in death [2]. Australia has one of the highest rates of skin cancer in the world. Over 1890 Australians die from skin cancer each year [3]. Melanoma is capable of deep invasion. The most dangerous characteristic of melanoma is that it can spread widely over the body via the lymphatic vessels and blood vessels. Thus, early diagnosis of melanoma is a key factor for prognosis of the disease.

Analysis of skin lesion images taken using dermoscopic imaging technique [4] is a commonly used method for diagnosis of skin cancer but, this method requires great deal of experience [5]. Due to the lack of reproducibility and subjectivity of human interpretation, the development of computerized image analysis techniques is of paramount importance. Segmentation is one of the most important and difficult task in computerized image analysis process. The accuracy of the subsequent steps highly depends on the success of image segmentation technique.

Segmentation of skin lesions is difficult because of the great variety of lesion shapes, sizes, and colors along with different types and textures of human skin. Other difficulties that make it a challenging task include, low contrast between the lesion and the surrounding skin, smooth transition between the lesion and the skin, reflections due to wrong illumination and artifacts such as skin texture, air bubbles and hair.

Image segmentation algorithms available in literature can be broadly classified into two categories: 1) discontinuity based segmentation 2) Similarity based segmentation, which can further be classified into thresholding [6], clustering [7], and region based approach [8]. Comparative analysis of these approaches in the area of skin lesion segmentation can be found in literature [9-11].

Active contour is a popular approach used to estimate boundaries in medical images. Two types of algorithms lie under this category: 1) parametric active contours [12] which adapt a deformable curve until it fits the object boundary. 2) Geometric active contours based on level set theory. Some of the active contour models need user intervention for initialization. Thus automatic approaches like gradient vector flow algorithm [13, 14] based on anisotropic diffusion [15] and robust algorithms like adaptive snakes and shape probability association model [16] are taking important place in literature.

Level set (LS) methods, as one of the automatic process approach have shown effective results for medical image segmentation. However, intensive computational requirements and regulation of controlling parameters make it a complex and time consuming method. To overcome such shortcomings, fuzzy clustering has been used to facilitate the LS segmentation for automatic segmentation of ultrasound, computed tomography and 
magnetic resonance imaging [17-19]. Fuzzy C mean (FCM) based thresholding [20] and LS algorithm are different computational models that have been applied individually for segmentation of dermoscopic images [21, 22]. Similarly, some work is done on using clustering for initial segmentation followed by further edge preserving and refining steps for border tracing of skin lesions [23].

We analyzed the advantages and limitations of these methods for skin lesion segmentation. The observations showed that FCM works well for making rough clustering of pixels which can further be used to get better threshold values for image segmentation. In addition to this, if the thresholded image is used as initial estimate for level set evolution, it can result in more accurate segmentation as compared to standard level set method [24,25] and region based active contours [26,27]. We, hereby presented a technique, based on combining these methods to achieve higher accuracy in segmentation of skin lesion images.

This paper introduces 3-class FCM based thresholding to be used for initializing the LS evolution and regulating the controlling parameters. Performance evaluation is done by comparing the diagnosis results of this method with three other segmentation methods i.e. FCM clustering [28], region based active contours [26, 29] and adaptive thresholding [11]. Results show that the proposed approach performs reasonably well for the segmentation of skin lesion images. The paper is organized as follows: Section 2 provides details of the proposed segmentation method. Section 3 discusses experimental results and Section 4 provides performance evaluation. Concluding remarks are given in Section 5.

\section{Methodology}

This section describes our technique for segmentation of skin cancer images. The main parts of our proposed algorithm are:

\subsection{Image Pre-Processing}

For skin lesion images there are certain extraneous artifacts such as skin texture, air bubbles, dermoscopic gel, presence of ruler markings and hair that make border detection a bit difficult. In order to reduce the effect of these artifacts on segmentation results, the images need to be pre-processed with a smoothing filter. We found that the best segmentation results were obtained using median filter with $7 \times 7$ mask to smooth the images before segmentation. Thus, the first step of the overall process is to get a filtered image.

\subsection{Fuzzy C-Mean Thresholding}

FCM clustering is used to partition $\mathrm{N}$ objects into $\mathrm{C}$ classes. In our method, $\mathrm{N}$ is equal to the number of pixels in the image i.e. $\mathrm{N}=\mathrm{N}_{\mathrm{x}} \times \mathrm{N}_{\mathrm{y}}$ and $\mathrm{C}=3$ for 3-class FCM clustering. The FCM algorithm uses iterative optimization of an objective function based on a weighted similarity measure between the pixels in the image and each of the c-cluster centers. A local extremism of the objective function indicates an optimal clustering of the input data. The objective function that is minimized is given by (1)

$$
Q=\sum_{i=1}^{C} \sum_{j=1}^{N}\left(u_{i j}\right)^{m}\left\|z_{j}-v_{i}\right\|^{2}
$$

where $z_{j} \in Z$ and $Z=\left\{z_{1}, z_{2}, z_{3} \ldots \ldots \ldots z_{N}\right\} \quad \& \quad v_{i} \in V$ where $V=\left\{v_{1}, v_{2}, \ldots v_{C}\right\} .\|*\|$ is a norm expressing the similarity between any measured data value and the cluster centre; $m \in[1, \infty]$ is a weighting exponent and can be any real number greater than 1 .

Calculations suggest that best choice of $\mathrm{m}$ is in the interval $[1.5,2.5]$, so $\mathrm{m}=2$ is used here as it is widely accepted as a good choice of fuzzification parameter. The fuzzy c-partition of given data set is the fuzzy partition matrix $\mathrm{U}=\left[u_{i j}\right]$ with $\mathrm{i}=1,2 \ldots \mathrm{C}$ and $\mathrm{j}=1,2,3 \ldots \mathrm{N}$, where $u_{i j}$ indicate the degree of membership of $\mathrm{j}^{\text {th }}$ pixel to $\mathrm{i}^{\text {th }}$ cluster. The membership functions are subject to satisfy the following conditions.

$$
\begin{gathered}
\sum_{i=1}^{C} u_{i j}=1 \text { for } \mathrm{j}=1,2,3, \ldots \mathrm{N} ; 0<\sum_{j=1}^{N} u_{i j}<N \text { for } \mathrm{i}= \\
1,2 \ldots . \mathrm{C} ; 0 \leq u_{i j} \leq 1
\end{gathered}
$$

The aim of FCM algorithm is to find an optimal fuzzy c-partition by evolving the fuzzy partition matrix $\mathrm{U}=$ $\left[u_{i j}\right]$ iteratively and computing the cluster centres. In order to achieve this, the algorithm tries to minimize the objective function Q (1) by iteratively updating the cluster centres and the membership functions using the following equations.

$$
\begin{gathered}
v_{i}=\left(\sum_{j=1}^{N}\left(u_{i j}\right)^{m}\right) /\left(z_{j} \sum_{j=1}^{N}\left(u_{i j}\right)^{m}\right) \\
u_{i j}=1 /\left(\sum_{k=1}^{C}\left(\frac{\left\|v_{i}-u_{j}\right\|}{\left\|v_{i}-u_{k}\right\|}\right)^{\frac{2}{m-1}}\right)
\end{gathered}
$$

After performing FCM clustering, finally each pixel is assigned to the cluster for which its membership value is maximum. Based on the intensity distribution obtained using histogram of the image, the threshold value is calculated by taking mean of maximum of cluster 1 and minimum of cluster 2 or maximum of cluster 2 and minimum of cluster 3 . This method of threshold selection takes into account the intensity distribution in the image. This choice helps in obtaining optimum threshold values for different images obtained under different conditions. The overall FCM thresholding algorithm is presented in Figure 1. The output of this stage is a binary image $(\mathrm{Bi})$ which has been used in the followed steps.

\subsection{Fuzzy C-mean Thresholding based Level Set Segmentation}

Segmentation of images by means of active contours is a well-established approach. Active contours [12] are used 
to detect objects in a given image using techniques of curve evolution. The basic idea is to evolve a curve, subject to constraints from a given image, for detecting objects in that image.

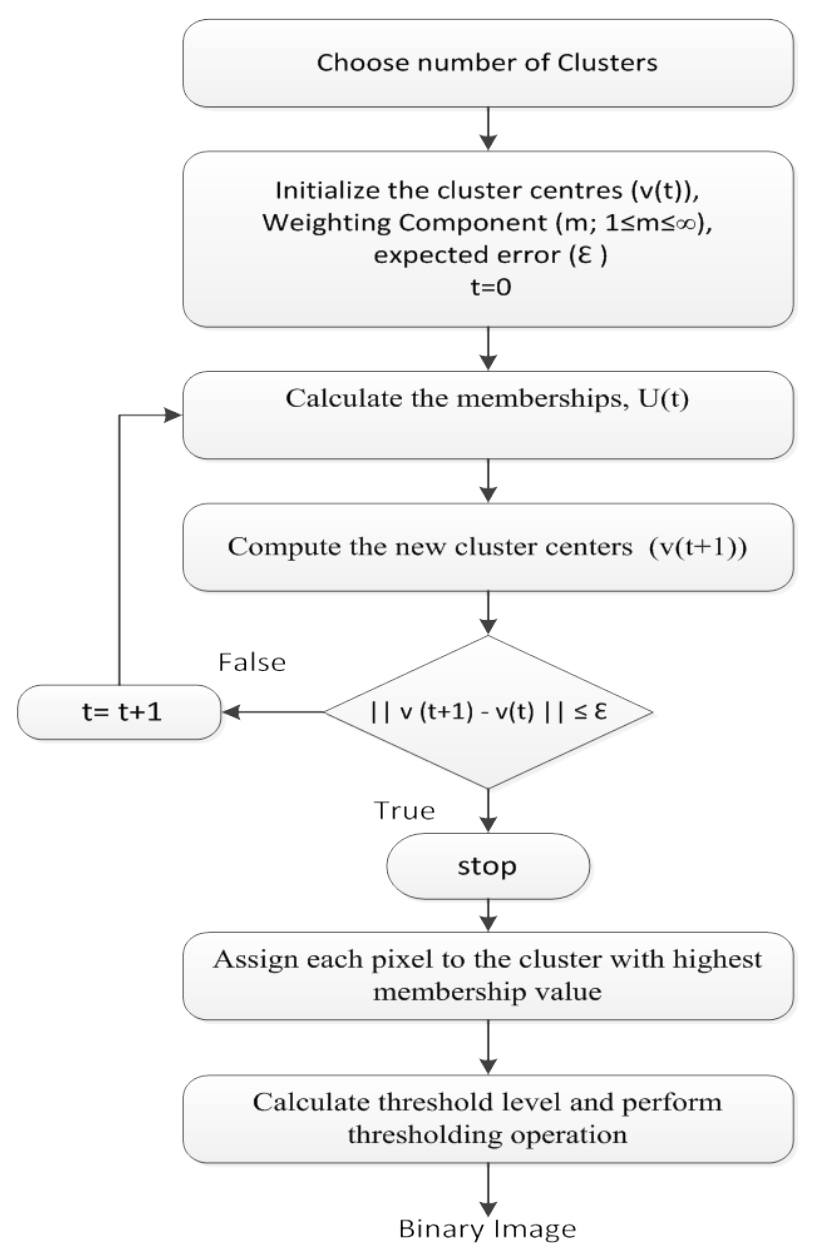

Figure 1. Algorithm for FCM thresholding.

In problems of curve evolution, level set methods have been extensively used. LS methods are established on dynamic implicit interfaces and partial differential equations (PDEs). In traditional LS formulation [25], the contours denoted by $\mathrm{C}$, are represented by the zero level set $\mathrm{C}(\mathrm{t})=\{(\mathrm{x}, \mathrm{y}) \mid \emptyset(\mathrm{t}, \mathrm{x}, \mathrm{y})=0)$ of a level set function $\emptyset$ $(\mathrm{t}, \mathrm{x}, \mathrm{y})$. The evolving equation of the level set function $\emptyset$ can be written in the following general form (4)

$$
\frac{\partial \emptyset}{\partial t}+F|\nabla \emptyset|=0
$$

which is called levels set equation. The function $\mathrm{F}$ is the speed function that represents the comprehensive forces, including the internal force from the interface geometry and the external force from image gradient or/and artificial momentums.

In order to stop the level set evolution near the optimal solution, the advancing force has to be regularized by an edge indication function $\mathrm{g}$. The edge indication function used here is given by (5)

$$
g=1 /\left(1+\left|\nabla I^{*}\right|^{2}\right)
$$

where $I^{*}$ is the filtered image. Traditional LS method is computationally intensive and has certain limitations like need of re-initialization of level set function to signed distance function for stable curve evolution [26]. Therefore, in this paper fast level set formulation has been used which was proposed by Li. et al. [24]. This method is computationally more efficient and can be implemented by using simple finite difference scheme. In order to segment the skin lesion image the overall iterative process for levels set evolution is given by (6).

$$
\emptyset^{j+1}=\phi^{j}+\tau\left[\xi\left(g, \phi^{j}\right)+\mu \xi\left(\phi^{j}\right)\right]
$$

where $\xi(g, \emptyset)=\pi \delta_{s}(\emptyset) \operatorname{div}\left(g \frac{\nabla \emptyset}{\|\emptyset\|}\right)+g v \delta_{s}(\emptyset)$ is the term for attracting $\emptyset$ towards the variational boundary and $\xi(\emptyset)=\left(\nabla^{2} \emptyset-\frac{\nabla \emptyset}{|\nabla \emptyset|}\right)$ is the penalty term that forces $\emptyset$ to approach the genuine signed distance function automatically.

This fast level set formulation proposed in [24] provides a benefit of flexible initialization where roughly obtained region from thresholding can be used to construct initial level set function. Taking advantage of this facility binary image (Bi) obtained from FCM thresholding algorithm, discussed in the previous section is used here for automatic initialization of the level set function $\emptyset$. The initial level set function is given as [18].

$$
\emptyset_{0}(x, y)=-4 \varepsilon(0.5-B i)
$$

where $\varepsilon$ is the regulator for dirac function [25] defined as follows:

$$
\delta_{s}(x)=\left\{\begin{array}{cc}
0, & |x|>\varepsilon \\
\frac{1}{2 \varepsilon}\left[1+\cos \left(\frac{\pi x}{s}\right)\right], & |x| \leq \varepsilon
\end{array}\right.
$$

Controlling parameters are also adaptively determined from the binary image (Bi) for regularizing the LS evolution process automatically. The weighting coefficient $\mu$ of penalty term $\xi(\emptyset)$ is taken as the ratio of area of on pixels in the binary image $(B i)$ to its perimeter pixels. The time step $\tau$ is taken as $0.2 / \mu$ so that $(\tau x \mu)$ remains smaller than 0.25 which is necessary to ensure stable evolution as found in [24]. $X$ is the coefficient of contour length for smoothness regulation and its value is taken here as $0.1 / \mu$. The value of $\lambda$ can be increased for accelerating the evolution process but it leads to smoother contours. Thus, care must be taken especially for skin lesion images, where over smoothened images may lose significant details about boundary of lesion, which is an important feature for correct diagnosis. The balloon force which determines the advancing direction 
and speed of the evolving curve is given as (9)

$$
v=-2(2 * \beta * B i-(1-\beta))
$$

where $\beta$ is the modulating argument which is taken here as 0.5 through experimental analysis. Figure 2 shows a systematic diagram of the proposed method. Each block represents different steps of the algorithm.

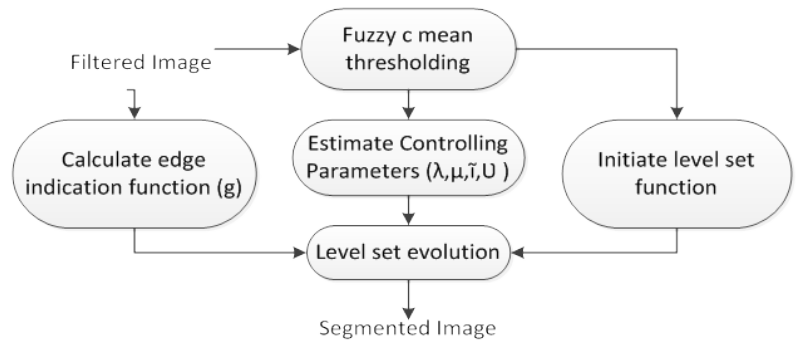

Figure 2. Flowchart of proposed algorithm.

\section{Experimental Results}

Segmentation results obtained using the proposed algorithm, FCM clustering, Adaptive Thresholding (AT) and Region based Active Contours (RBACs) are presented in Figures 3-7 for some of the skin lesion images. While presenting results, we tried to present images having different common problems of dermoscopic images which can badly affect the segmentation process. Figure $\mathbf{3}$ shows an image of dysphasic nevi having poor illumination and uneven boundary of lesion. Figure 4 represents melanoma lesion with uneven border and hair on it. Figure 5 is melanoma lesion with smooth transaction between lesion and skin. Figure $\mathbf{6}$ is a melanoma lesion present on a skin with spots and redness effect. Figure 7 is a benign lesion surrounded by a lot of hair.

FCM clustering and AT provide rough segmentation but cannot track the boundary exactly. Region based active contours provided results comparable to proposed method in some cases but this method cannot track the border in the presence of many hair (see Figure 7(f)) or spotty skin (see Figure 6(f)). Similarly, it missed the exact boundary in the case where there is a smooth transaction between lesion and skin (see Figure 5(f)).

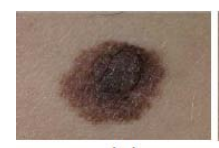

(a)

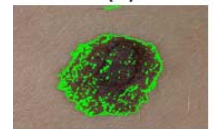

(d)

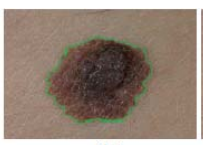

(b)

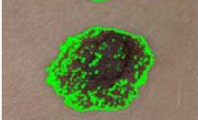

(e)

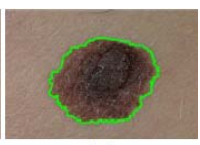

(c)

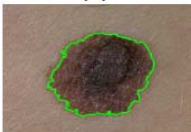

(f)
Figure 3. Segmentation results of dysplastic nevi (a) original image (b) Ground truth by expert (c) Proposed method (d) FCM clustering (e) AT (f) RBACs.

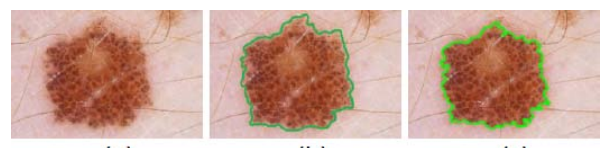

(a)

(b)

(c)

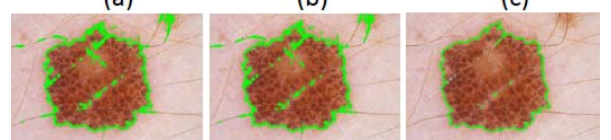

(d)

(e)

(f)

Figure 4. Segmentation results of melanoma (a) original image (b) Ground truth by expert (c) Proposed method (d) FCM clustering (e) AT (f) RBACs.

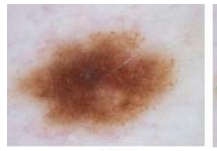

(a)

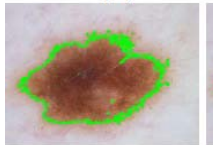

(d)

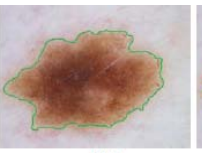

(b)

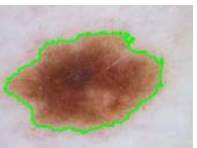

(c)

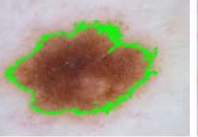

(e)

Figure 5. Segmentation results of melanoma (a) original image (b) Ground truth by expert (c) Proposed method (d) FCM clustering (e) AT (f) RBACs.

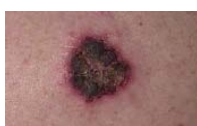

(a)

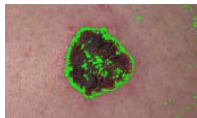

(d)

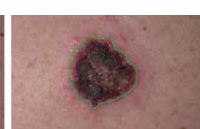

(b)

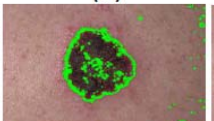

(e)

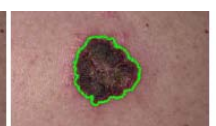

(c)

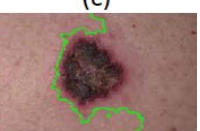

(f)

Figure 6. Segmentation results of melanoma (a) original image (b) Ground truth by expert (c) Proposed method (d) FCM clustering (e) AT (f) RBACs.

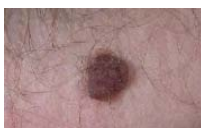

(a)

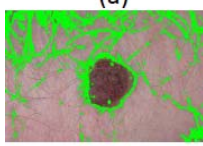

(d)

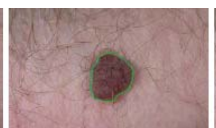

(b)

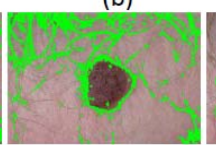

(e)

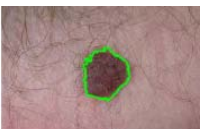

(c)

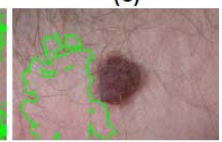

(f)

Figure 7. Segmentation results of benign lesion (a) original image (b) Ground truth by expert (c) Proposed method (d) FCM clustering (e) AT (f) RBACs.

Analysis of segmentation results showed accuracy of proposed method even in the presence of all these artifacts.

\section{Evaluation of Segmentation Results}

The objective evaluation of segmentation algorithms on a 
large set of clinical data is one of the important steps toward establishing validity and clinical applicability of an algorithm. Thus, in order to evaluate the efficiency of the proposed method a performance comparison is provided with three of the well-known segmentation methods used here for segmentation of same collection of skin lesion images. The metric used here for measurements is based on pixel-by-pixel comparison of pixels enclosed in the segmented result (SR) and the ground truth result (GT) from expert. First, binary images are constructed for each boundary, where a pixel is considered non-zero if it lies inside the boundary and zero otherwise. Error value shows the root mean square error by taking ground truth image as standard segmentation result. Other evaluation parameters are calculated as follows:

Hammoude distance (HM): This metric makes a pixel by pixel comparison of the pixels enclosed by the two boundaries:

$$
\mathrm{HM}(\mathrm{SR}, \mathrm{GT})=\frac{\#(\text { SR UGT })-\#(\text { SR } \cap G T)}{\#(\text { SR UGT })}
$$

This well-known metric takes into account two types of error; pixels classified as lesion by automatic segmentation that were not classified as such by medical expert and pixels classified as lesion by medical expert that were not classified as such by automatic segmentation. The Hammoude distance gives equal importance to both types of errors. However, from a clinical point of view, the 2nd type of error is more important since the lesion pixels should never be missed by the automatic diagnostic system. Therefore, separate metrics should be used to take into account the two types of error.

True detection rate (TDR): This metric measure the rate of pixels classified as lesion by both the automatic and the medical expert segmentation. Higher TDR shows better performance of segmentation method.

$$
\mathrm{TDR}(\mathrm{SR}, \mathrm{GT})=\frac{\#(\mathrm{SRnGT})}{\#(\mathrm{GT})}
$$

False Positive Error (FPE): This metric determines the rate of pixels assigned as lesions by the segmentation method that were not assigned as lesion by the medical expert. Lower the value of FPE better is the performance of respective segmentation method.

$$
\mathrm{FPE}(\mathrm{SR}, \mathrm{GT})=\frac{\#(\mathrm{SR} \cap \mathrm{G} \overline{\mathrm{T}})}{\#(\mathrm{GT})}
$$

False Negative Error (FNE): It determines the rate of pixels categorized as lesions by the medical expert that were not assigned as lesion by the automatic segmentation:

$$
\mathrm{FNE}(\mathrm{SR}, \mathrm{GT})=1-\frac{\#(\mathrm{SRnGT})}{\#(\mathrm{GT})}
$$

Table 1 shows the comparative results. The database used for analysis comprised of 238 dermoscopic and clinical view lesion images which were collected from various sources but most images were obtained from Sydney Melanoma Diagnostic Centre, Royal Prince Alfred Hospital. The segmentation results were compared with the reference images (ground truth) and average of segmentation scores are presented here for each method.

Table 1. Results of Segmentation Methods. The values in bold correspond to the best performance.

\begin{tabular}{cccccc}
\hline \multirow{2}{*}{ Method } & \multicolumn{5}{c}{ Evaluated Parameters } \\
\cline { 2 - 6 } & FNE (\%) & $\begin{array}{c}F P E \\
(\%)\end{array}$ & $\begin{array}{c}\text { TDR } \\
(\%)\end{array}$ & $\begin{array}{c}H M \\
(\%)\end{array}$ & $\begin{array}{c}\text { Error } \\
\%)\end{array}$ \\
\hline $\begin{array}{c}\text { FCM } \\
\text { Clustering }\end{array}$ & 13.75 & 6.52 & 86.25 & 34.8 & 34.31 \\
\hline RBACs & 11.72 & 9.08 & 88.28 & 37.1 & 36.47 \\
\hline AT & 13.14 & 6.95 & 86.86 & 35.2 & 34.94 \\
\hline $\begin{array}{c}\text { FCM } \\
\text { thresholding } \\
\text { based LS }\end{array}$ & $\mathbf{7 . 3 4}$ & $\mathbf{4 . 6 6}$ & $\mathbf{9 2 . 6 6}$ & $\mathbf{1 1 . 3}$ & $\mathbf{1 5 . 1 9}$ \\
\hline
\end{tabular}

It is evident from the results that the proposed method has shown reasonably better performance as compared to other methods. Region based active contour method has shown low FNE and higher TDR as compared to FCM clustering, and adaptive thresholding but it has quite high false positive error, which makes it susceptible of declaring benign lesions as melanoma. On the basis of our analysis, we believe that the proposed method can show promising results for lesion segmentation in a computer aided diagnosis system.

\section{Conclusions}

In this paper, a segmentation algorithm is presented for skin lesion detection. It combines the advantages of clustering, thresholding and level set method, for getting more accurate segmentation results. The proposed method showed reasonably good accuracy for segmentation of skin lesion images with an average true detection rate of $92.6 \%$ and quite reduced false positive and false negative error i.e. $4.66 \%$ and $7.34 \%$ respectively. Comparative analysis proved that it works well even in the presence of different artifacts present in skin images. Keeping an eye on the importance of tissue and cell level diagnosis of skin cancer, this method can provide a basis for segmenting histo-pathological images as well.

\section{REFERENCES}

[1] R. Siegel, et al., "Cancer statistics, 2011”, CA: A Cancer Journal for Clinicians, Vol. 61, No. 4, 2011, pp. 212-236. doi:10.3322/caac.20121

[2] Society, A.C., Cancer Facts \& Figures 2012, http://www.cancer.org/acs/groups/content/epidemiologys urveilance/documents/document/acspc-031941.pdf 2012.

[3] “Causes of Death 2010,” C.W.O. Australia, Editor, Aus- 
tralian Bureau of Statistics, Canberra, Australia.

[4] G. Argenziano and H.P. Soyer, "Dermoscopy of pigmented skin lesions, a valuable tool for early diagnosis of Melanoma,” The Lancet Oncology, Vol. 2, No. 7, 2001, pp. 443-449. doi:10.1016/S1470-2045(00)00422-8

[5] D. Piccolo, et al., "Dermoscopic Diagnosis by A Trained Clinician vs. A Clinician with Minimal Dermoscopy Training vs. Computer-aided Diagnosis of 341 Pigmented Skin Lesions: A Comparative Study,” British Journal of Dermatology, Vol. 147, No. 3, 2002, pp. 481-486. doi:10.1046/j.1365-2133.2002.04978.x

[6] S. Ben Chaabane, et al., "Color Image Segmentation Using Automatic Thresholding and the Fuzzy C-means Techniques", in Proceedings 14th IEEE Mediterranean Electro technical Conference, 2008, pp. 857-861.

[7] L. Dongju and Y. Jian. , “Otsu Method and K-means,” in Proceedings Ninth International Conference on Hybrid Intelligent Systems, China, 2009, pp. 344-349.

[8] M. Emre Celebi, et al., "Border Detection in Dermoscopy Images Using Statistical Region Merging”, Skin Research and Technology, Vol. 14, No. 3, 2008, pp. 347-353. doi:10.1111/j.1600-0846.2008.00301.x

[9] M. E. Celebi, G. S. H. Iyatomi and W. V. Stoecker, "Lesion Border Detection in Dermoscopy Images," Computerized Medical Imaging \& Graphics, Vol. 33, 2009, pp. 148-153.

[10] T. Mendonca, et al., "Comparison of Segmentation Methods for Automatic Diagnosis of Dermoscopy Images”, in Proceedings of 29th Annual International Conference of the IEEE Engineering in Medicine and Biology Society, 2007, pp. 6572-6575.

[11] M. Silveira, et al., "Comparison of Segmentation Methods for Melanoma Diagnosis in Dermoscopy Images," IEEE Journal of Selected Topics in Signal Processing, Vol. 3, No. 1, 2009, pp. 35-45. doi:10.1109/JSTSP.2008.2011119

[12] Isard, A. B. A. M., Active Contours 1998: Springer Verlag.

[13] Mahmoud, M. K. A. and A. Al-Jumaily, "Segmentation of Skin Cancer Images Based on Gradient Vector Flow Snake,” in Proceedings of 2011 International Conference on Mechatronics and Automation, 2011, pp. 216-220. doi:10.1109/ICMA.2011.5985659

[14] Bulent Erkol, R. H. M., R. Joe Stanley, William V. Stoecker and Erik Hvatum, "Automatic Lesion Boundary Detection in Dermoscopy Images Using Gradient Vector Flow Snakes,” Skin Research and Technology, Vol. 11, No. 1, 2005, pp. 17-26. doi:10.1111/j.1600-0846.2005.00092.x

[15] P. Perona, and J. Malik, "Scale-space and Edge Detection Using Anisotropic Diffusion," IEEE Transactions on Pattern Analysis and Machine Intelligence, Vol. 12, No. 7, 1990, pp. 629-639. doi:10.1109/34.56205

[16] J. C. Nascimento, et al., "Adaptive Snakes Using the EM Algorithm," IEEE Transactions on Image Processing,
Vol. 14, No. 11, 2005, pp.1678-1686. doi:10.1109/TIP.2005.857252

[17] M. N. M. Babu, V. K. Hanmandlu, M. Vasikarla, S., "Histo-pathological Image Analysis Using OS-FCM and Level Sets", in Proceedings of IEEE 39th Applied Imagery Pattern Recognition Workshop 2010, pp. 1-10.

[18] Li, B. N. et al., "Integrating Spatial Fuzzy Clustering with Level Set Methods for Automated Medical Image Segmentation”, Computers in Biology and Medicine, Vol. 41, pp. 1-10, 2011. doi:10.1016/j.compbiomed.2010.10.007

[19] B. N. Li, et al., "Integrating FCM and Level Sets for Liver Tumor Segmentation,” in Proceedings 13th Int. Conference on Biomedical Engineering, Singapore, 2009, pp. 202-205.

[20] Aja-Fernandez et al., "Soft Thresholding for Medical Image Segmentation," in Proceedings 32nd Annual International Conference of the IEEE Engineering in Medicine and Biology Society, Argentina, 2010, pp. 4752-4755.

[21] S. Sookpotharom, "Border Detection of Skin Lesion Images Based on Fuzzy C-Means Thresholding," in Proceedings of 3rd Int. Conference on Genetic and Evolutionary Computing, China, 2009, pp. 777-780.

[22] M. Silveira and J. S. Marques, "Level Set Segmentation of Dermoscopy Images,” presented at 5th IEEE International Symposium on Biomedical Imaging: From Nano to Macro, May 14-17, 2008.

[23] M. Kamali and G. Samei, "Border Preserving Skin Lesion Segmentation," in Proceedings of SPIE 6915, Medical Imaging 2008: Computer-Aided Diagnosis, 2008.

[24] L. Chunming, et al., "Level Set Evolution without Re-initialization: A New Variational Formulation," in Proceedings of IEEE Computer Society Conference on Computer Vision and Pattern Recognition, San Diego, 2005, pp. 430-436.

[25] S. Osher and R. Fedkiw, "Level Set Methods and Dynamic Implicit Surfaces2002,” New York: Springer-Verlag.

[26] T. F. Chan and L. A. Vese, "Active contours without edges,” IEEE Transactions on Image Processing, Vol. 2, pp.266-277, 2001. doi:10.1109/83.902291

[27] V. Caselles, R. Kimmel, and G. Sapiro, “Geodesic Active Contours”, International Journal of Computer Vision, Vol. 22, No. 1, 1997, pp. 61-79. doi:10.1023/A:1007979827043

[28] Huiyu, Z., et al., "Anisotropic Mean Shift Based Fuzzy C-Means Segmentation of Dermoscopy Images,” IEEE Journal of Selected Topics in Signal Processing, Vol. 3, No. 1, 2009, pp. 26-34. doi:10.1109/JSTSP.2008.2010631

[29] Q. Abbas, I. Fondón and M. Rashid, "Unsupervised Skin Lesions Border Detection via Two-dimensional Image Analysis," Computer Methods and Programs in Biomedicine, Vol. 104, No. 3, 2011, pp. 1-15. doi:10.1016/j.cmpb.2010.06.016 